\title{
Le coton biotechnologique au Burkina Faso : trajectoire d'une innovation dans un contexte de développement
}

\author{
Biotechnology Cotton in Burkina Faso: Trajectory of an Innovation in a \\ Development Context
}

\author{
Eveline M.F.W. Compaoré Sawadogo ${ }^{1}$ \\ ${ }^{1}$ Institut de l'Environnement et de la Recherche Agricole (INERA), Burkina Faso, compeve@yahoo.fr
}

RÉSUMÉ. Cet article retrace la trajectoire de l'innovation du coton biotechnologique, connu sous le nom de Bacillus thuringiensis (Bt), au Burkina Faso. II soutient que l'introduction de ce coton biotechnologique dans le système d'innovation agricole au Burkina Faso a entraîné un déplacement du contrôle de pouvoir des acteurs nationaux (industries, chercheurs, agriculteurs, Etat, société civile), en faveur d'acteur étranger (Monsanto) qui utilise sa position pour maximiser son gain économique aux dépens d'un grand nombre de petits agriculteurs et du système national de recherche agricole. Cet article a utilisé le concept analytique de la théorie de l'acteur réseau, un concept qui provient de l'anglais Actor Network Theory (ANT) (CALLON 1986). Elle éclaire les complexités en reliant les acteurs. La méthode ethnographique, y compris l'entretien et l'observation a été utilisée pour reconstituer le système d'innovation de ce coton biotechnologique.

ABSTRACT. This article traces the innovation of biotechnology cotton, known as Bacillus thuringiensis (Bt), in Burkina Faso. It argues that the introduction of this cotton into the agricultural system of the country has led to a shift in the control of the power of the national actors (industries, researchers, farmers, state, civil society) for foreign actor (Monsanto), which uses its position to maximize its economic gain at the expense of a large number of small farmers and the national agricultural research system. To define the network of actors, this paper uses the analytical concept of Actor Network Theory (ANT) (CALLON 1986). It is a unique approach that sheds light on the complexities of connecting actors. The ethnographic method, including interviews and observations were used to reconstruct the innovation system of this biotechnological cotton.

MOTS-CLÉS. trajectoire d'innovation, coton biotechnologique, échec.

KEY WORDS. innovation trajectory, biotechnological cotton, failure.

\section{Introduction}

$\mathrm{Au}$ Burkina Faso, environ 90\% de la population est engagée dans l'agriculture vivrière (GRAY 2008). La production du coton constitue le second pilier de l'économie du pays (ISAAA 2006, TIENDREBEOGO 2011). Le revenu du coton contribue aux moyens de subsistance de plus de 3 millions de personnes représentant environ $20 \%$ de la population, les aidant à soutenir leur famille et à envoyer leurs enfants à l'école. Cette principale culture d'exportation, qui a elle seule englobe $60 \%$ des recettes d'exportation du pays (CNRST 2007, CES 2011), est vulnérable aux sécheresses, fréquentes et aux dommages causés par les insectes, rendant le coton extrêmement dépendant de l'usage des insecticides. L'utilisation croissante des pesticides dans le long terme a entraîné les résistances des insectes et ce depuis les années 1990. Les contraintes de productivité et de résistance aux insectes, dans le système social et technique du coton conventionnel, d'une part, et les promesses des biotechnologies développées par Monsanto ${ }^{1}$ pour résoudre ces problèmes, d'autre part constituent de prime abord un contexte favorable à l'adoption du coton biotechnologique au Burkina Faso. Le coton biotechnologique ou encore coton biotechnologique contraste avec les objets traditionnels de la sociologie de la

\footnotetext{
${ }^{1}$ Pour Monsanto, sa mission est d'utiliser les OGM pour produire plus tout en conservant plus de ressources environnementales. Monsanto est considéré comme une société de recherche agricole. II reste selon sa vision l'une des principales industries de pointe en biotechnologie agricole dans le monde. À cet égard, Monsanto se présente comme un leader en biotechnologie agricole en termes d'expertise, de compétitivité et d'affaires. 
traduction, car il soulève de nombreuses questions éthiques rendant son transfert plus complexe dans le contexte de pays en développement. En effet, selon la théorie de l'innovation, l'interaction entre le contexte social, y compris le marché, les acteurs et la technologie elle-même est essentielle pour comprendre comment le processus d'introduction de cette nouvelle technologie repose sur une dynamique du réseau d'acteurs humains et non humains (CALLON 1986, CALLON 1991).

Cet article fait valoir que l'introduction du coton biotechnologique dans le système agricole au Burkina Faso a entraîné une dévaluation des savoirs scientifiques conventionnels avec pour conséquence le déplacement du contrôle du pouvoir des mains des acteurs nationaux (recherche, Etat, les industries nationales, les producteurs et les sociétés rurales) en faveur des intérêts et du contrôle d'un acteur étranger privé (Monsanto). Ce dernier dans le processus de construction du réseau d'acteur a agi pour maximiser son gain économique ${ }^{2}$ aux dépens d'un grand nombre de petits agriculteurs et du système national de recherche agricole, entrainant ainsi, après 10 ans de diffusion de cette variété OGM, sa suspension en 2016 au Burkina Faso. La prise en compte des conditions dans lesquelles cette technologie s'est imposée dans un pays en développement et qui a échoué remet en question la vision optimiste et abstraite du paradigme de développement axé sur le rôle de la science et de la technologie dans le développement. Plus précisément, la validité de l'affirmation selon laquelle les interventions scientifiques et technologiques produiraient en soi un développement socio-économique n'est pas soutenue. L'accent est mis sur l'histoire du processus d'introduction du coton biotechnologique et son développement en matière d'identification et de description des différents acteurs et de leur implication dans le système d'innovation du coton Bt. Cela aidera à comprendre comment la transition du coton conventionnel au coton biotechnologique a eu lieu au Burkina Faso et comment le système social et technique du coton Bt a été construit.

Cet article utilise le concept analytique de la théorie de l'Acteur Réseau ou encore en anglais, Actor Network Theory (ANT) pour retracer la trajectoire ou le diagramme du système d'innovation du coton Bt. En effet, cette approche constructiviste suppose que les événements ou les innovations sont mieux compris dans l'action plutôt que de les donner des attributs abstraits (CALLON 1986, AKRICH et al., 1988 ; CALLON 1991, LATOUR 2005). C'est un cadre théorique qui éclaire les complexités en reliant les personnes, les artefacts, les institutions et les organisations. Plus explicitement, cette théorie stipule que la réalité ou la forme des choses dépendent de leur participation aux relations entrelacées. L'acteurréseau est le concept central dans ANT. Cependant, l'ANT est complètement processuel, ce qui signifie qu'il n'y a aucune garantie que les acteurs du réseau resteront les mêmes tout au long du processus de construction du réseau et de son fonctionnement. De toute évidence, ANT : « se compose de quatre moments qui peuvent en réalité se chevaucher. Ces moments constituent les différentes phases d'un processus général appelé traduction, au cours duquel l'identité des acteurs, la possibilité d'interaction et les marges de manœuvre sont négociées et délimitées » (CALLON et BLACKWELL 2007 : 59). Dans ce cadre, il peut envisager des stratégies explicites pour relier les différents éléments en un réseau afin qu'ils forment un ensemble cohérent. Les théoriciens de l'ANT supposent également que les réseaux de relations sociales ne sont pas harmonieux et peuvent contenir des conflits. ANT suppose donc que toutes les entités d'un réseau peuvent et doivent être décrites dans les mêmes termes. Il y a donc de bonnes raisons de le considérer comme un cadre utile pour l'analyse du système d'innovation du coton Bt. En fait, pour ce qui concerne les processus de traduction, de la problématisation, de l'intéressement, de l'enrôlement et de la mobilisation des alliés, la conceptualisation de l'ANT par Callon constitue une contribution importante dans la présente étude. En effet, ces processus peuvent être observés dans le

\footnotetext{
${ }^{2}$ Selon cette déclaration d'un producteur de coton "Nous sommes opposés aux OGM pour plusieurs raisons évidentes...L'augmentation du coût des semences, de 1600 FCFA [24 Euros / 34 USD] par hectare pour les semences conventionnelles l'an dernier à 54000 FCFA [82 Euros / 115 USD] par hectare pour les semences GM cette année, n'est pas accompagnée d'une augmentation du rendement Comme promis. Pire encore, le coton Bt produit moins de graines que la variété conventionnelle, et est donc deux fois plus léger pour la même production de fibres. Ainsi, les paysans payés par le poids de leur récolte sont les perdants au profit de Monsanto ... "
} 
cas de la technologie du coton biotechnologique au Burkina Faso et permet de voir dans quelle mesure Monsanto, les chercheurs, les producteurs de coton, les décideurs politiques, les activistes, le coton biotechnologique lui-même, sont tous entrés dans un réseau de relations à travers différentes séries de traduction.

\section{Encadré. Méthodologie de collecte des données}

Les méthodes ethnographiques, y compris les entretiens semis ouverts approfondis avec les décideurs politiques, les agriculteurs du coton au Centre et à l'Ouest du pays, les entretiens avec les représentants de Monsanto au Burkina, les acteurs de la société civile et des chercheurs ont été menées. Ces entretiens ont duré entre 60 et $120 \mathrm{mn}$. Au total 60 entretiens sur les processus organisationnels ont été analysés en plus d'une grande quantité de littérature grise sur le coton publiée entre 1961 et 2016.

L'analyse de ces données a démontré que le débat sur le transfert ou le développement interne de la technologie dans les pays en développement ne doit pas masquer la compréhension des formes de contrôle politique (tant sur le plan national qu'international) que la science et la technologie apportent avec elles. En général, la plupart des avantages sont limités à une poignée d'acteurs, à savoir les grands acteurs industriels et Monsanto, tandis que le grand nombre des agriculteurs qui ont besoin d'une technologie sûre qui améliorerait leurs conditions de vie restent dans le désespoir. Les progrès scientifiques et technologiques ne sont pas nécessairement une panacée pour les problèmes de développement car, dans certains cas, ces progrès peuvent aggraver la pauvreté. Tout débat sur le rôle de la science et de la technologie au service du développement doit donc tenir compte de la structure des dispositifs du réseau d'acteurs, des motivations et du processus par lequel les différents acteurs intègrent le réseau (COMPAORE 2015).

Dans la première partie, l'article rappelle le contexte qui a motivé l'adoption du coton biotechnologique au Burkina Faso à travers une description des problèmes de développement dans différents secteurs en mettant l'accent sur les difficultés classiques de la culture du coton dans le secteur agricole, et la nécessité d'adopter une nouvelle technologie.

Ensuite, la deuxième partie de l'article explique les conditions d'émergence et de stabilisation du système acteurs et des cadres institutionnels qui ont adopté le coton biotechnologique au Burkina.

Enfin en conclusion, l'article met en discussion l'échec du coton biotechnologique en abordant la manière dont la transition s'est faite par la construction de réseaux d'acteurs et la construction d'attentes sur le développement de la technologie.

\section{L'émergence du coton biotechnologique dans un contexte de problèmes de développement persistants}

La productivité de la culture du coton a diminué après le milieu des années 1980 et a encore diminué au cours des années 1990 (CNRST 2007). Par conséquent, les producteurs de coton, les industries du coton et le gouvernement se trouvaient tous confrontés à des difficultés liées à la lutte contre les ravageurs du coton afin d'accroître leur productivité et de maintenir le rôle de premier plan du pays dans la culture du coton en Afrique. Il est devenu impératif que les chercheurs cherchent des solutions efficaces. (SCHWARTZ 1999, CNRST 1994, 1995, 2007, CES 2011). 
D'autres problèmes persistants, tels que la dégradation des sols et les variations climatiques combinées à un manque crucial de précipitations ont entravé le développement de la croissance du coton au Burkina Faso (CNRST 94). Le coton conventionnel, cultivé depuis l'époque coloniale et développé à la période d'après-indépendance, n'était plus une perspective très prometteuse (SCHWARTZ 1997, GRAY 2008).

À partir des années 1980, on avait soutenu qu'un « secteur spécifique de la recherche cotonnière était nécessaire pour augmenter sa productivité » (CNRST 2007 : 26). L'INERA, le centre national de recherche agronomique, a été formé et a mené des recherches intensives grâce à la création d'un programme spécifique de recherche sur le coton (CNRST 2007, GRAY 2008). Ce faisant, ils espèrent remédier aux catastrophes liées à la résistance aux insectes auxquelles sont confrontés les producteurs de coton au Burkina Faso (CES 2011). En effet, ces problèmes ont découragé de nombreux acteurs impliqués dans la culture du coton, en particulier les cultivateurs de coton qui perdaient espoir quant à l'avenir de leur récolte commerciale. Malgré les efforts importants du programme de recherche sur la culture du coton, le Burkina Faso a donc été témoin, dans les années 1990, d'une baisse de sa production de coton à près de $90 \%$, tout comme ses pays voisins, le Mali et la Côte d'Ivoire (BAFFES 2007). Au Burkina Faso, comme dans d'autres pays d'Afrique de l'Ouest, la culture du coton est devenue de plus en plus dépendante des traitements insecticides pour lutter contre les parasites. Par exemple, six à huit traitements ont été recommandés afin d'obtenir une récolte satisfaisante. Cet état de fait a réduit la compétitivité du Burkina Faso sur le marché mondial (CNRST, 2007). Les agriculteurs ainsi que les chercheurs et les industries ont commencé à chercher une solution pour rehausser la productivité du coton (SCHWARTZ 1991, 1995, GRAY 1999, 2005, 2008, ROBERTS 1995). Le problème des insectes a eu une incidence non seulement sur la quantité, mais aussi sur la qualité de la production de coton conventionnel qui a affecté la compétitivité du pays sur le marché mondial du coton.

Par conséquent, bien que très contestée, la biotechnologie était considérée comme une des solutions qui augmenterait la production de coton et permettrait au Burkina Faso d'être compétitif sur le marché mondial. Cependant, certains auteurs affirment tous que les cultures des OGM sont dangereuses et que leurs effets secondaires ont d'énormes répercussions sur la sécurité humaine, animale et environnementale (SHIVA 2003, SMITH 2005, QAYUM et SAKKHARI 2005, ROBIN 2008). En revanche, Illy et Zangré pensent qu'il est " indéniable que OGM constitue aujourd'hui un enjeu économique important » (ILLY et ZANGRE, 2003 : 6). Pour eux, la biotechnologie est un des moyens pour le Burkina Faso de développer sa stratégie économique.

Monsanto, le propriétaire du gène du coton biotechnologique, est une entreprise relativement nouvelle. Pour Monsanto, sa mission est de produire plus tout en conservant plus de ressources environnementales (ISAAA 2006 ; EZEZIKA et al., 2012). C'est une entreprise qui conduit des recherches en biotechnologie la plus connue, axée sur l'agriculture. L'histoire de la biotechnologie dans l'agriculture montre que Monsanto est impliqué dans la recherche agricole biotechnologique depuis 1973 (GROSS 2007, EZEZIKA et al., 2012). Etant le propriétaire du gène de la graine de coton biotechnologique $(\mathrm{Bt})$, il fait largement sa promotion dans les pays en développement comme le Burkina Faso. Monsanto demeure l'une des principales industries de pointe en biotechnologie agricole dans le monde (JAMES 2009, ISAAA 2012, CES 2011, TRAORE 2011). À cet égard, Monsanto se présente comme un leader en biotechnologie agricole en termes d'expertise, de compétitivité et d'affaires (BORLAUG 2000, GROSS 2007, TRAORE 2011). 


\section{Les mécanismes institutionnels qui ont présidé à l'adoption de l'innovation coton biotechnologique au Burkina Faso}

La rencontre lors d'une table ronde au Cameroun en 1999 sur la biotechnologie dans laquelle le Burkina Faso a participé marque une étape décisive dans l'adoption du coton biotechnologique par les acteurs impliqués dans la production de coton au Burkina Faso. Le directeur de l'Agence Nationale de la Valorisation des Résultats de la Recherche (ANVAR) a été chargé par le gouvernement de participer à cette table ronde. Ce fut l'opportunité qui a permis d'établir le premier contact entre Monsanto, qui a fait une présentation sur le coton Bt, et le directeur de l'ANVAR, qui a fait une présentation sur les biotechnologies traditionnelles et le problème de la résistance des insectes du cotonnier au Burkina Faso. La relation entre les activités de recherche et les problèmes agricoles, particulièrement en ce qui concerne spécifiquement le coton, a été présentée par le directeur de l'ANVAR et les solutions ont été proposées par Monsanto à travers sa présentation sur le coton biotechnologique qui permet de lutter contre les insectes. Ces présentations ont mis en évidence des intérêts apparemment réciproques de ces deux acteurs (Monsanto et les acteurs nationaux). Dans l'histoire du coton biotechnologique au Burkina Faso, cette table ronde peut être considérée comme le point de départ, le début d'un long processus de négociations entre Monsanto et les différents acteurs locaux au Burkina Faso. Un participant à cette table ronde confirme :

«Après notre présentation, Monsanto nous a rencontrés pour discuter. Il était intéressé par le Burkina Faso. Donc Monsanto est déjà un acteur, un acteur principal. "Moi", je représentais la recherche, mais j'étais l'interface, parce que j'étais là-bas sous l'Agence [ANVAR] pour l'utilisation des résultats de la recherche. Puis nous étions deux acteurs. Il [l'officier de Monsanto] s'est dit intéressé par le Burkina Faso, le Mali et la Côte d'Ivoire. J'ai dit qu'il n'y avait pas de problème, mais parce que je suis un représentant du pays, je ne peux prendre aucune décision d'une telle importance. Mais je suggère qu'il puisse venir au Burkina, nous allons organiser un forum national où il va présenter son coton Bt. Et cela s'est passé en $2000 »$ (interview).

La conférence du Cameroun en 1999 a donc abouti à la manifestation d'un intérêt de Monsanto pour le transfert de sa technologie au Burkina Faso. Le directeur de l'ANVAR a indiqué l'intérêt du pays pour cette technologie. En tant que chercheur et directeur national de l'institut, son avis est susceptible d'être partagé par l'institution qu'il représente. Ce fut donc, à l'issue de la table ronde, le début d'un long processus de construction du système d'acteurs. Ce système est composé du coton conventionnel, les producteurs de coton, l'industrie cotonnière, les chercheurs sur le coton, la société civile et l'État. Les raisons pour lesquelles ces personnes sont concernées sont décrites ci-dessous.

\section{Définition des acteurs et leur place dans la trajectoire d'innovation Bt}

\subsection{Les producteurs de coton}

Les agriculteurs produisent du coton et sont directement touchés par les problèmes qui ont touché la récolte des années 1980. Ils sont confrontés à des difficultés croissantes liées à la lutte contre les ravageurs et à la dégradation des sols, combinée à des changements climatiques. Cette situation grave a découragé de nombreux agriculteurs, mettant en péril leurs espoirs et émettant des doutes quant à la poursuite ou non de la culture du coton (TIENDREBEOGO 2011). Les agriculteurs dans ce voyage allégorique de la croissance du coton dépensaient de plus en plus d'argent pour les soins du coton. En outre, ils ont été accusés par l'industrie et les chercheurs de ne pas utiliser correctement les insecticides et de détourner l'utilisation d'insecticides du coton vers d'autres cultures ou de les vendre sur le marché local. Il fallait donc s'attendre à ce que les agriculteurs soient intéressés à une technologie qui va les soulager et les permettre de tirer davantage de profits de la culture du coton. 


\subsection{Les industries du coton}

Les industries du coton sont des éléments clés du processus de croissance du coton au Burkina Faso. Elles ont un monopole sur les matériaux nécessaires à la production et à la transformation du coton. Leur singularité repose sur le fait que sans elles, les agriculteurs ne pouvaient pas se permettre de cultiver du coton à leurs propres frais. Elles fournissent aux agriculteurs des prêts, des insecticides, des produits chimiques, des outils, etc. pour la culture du coton. En outre, elles achètent tout le coton des agriculteurs afin de le traiter pour le marché mondial. La commercialisation du coton ne dépend donc pas des agriculteurs, mais des industries. La forte dépendance du coton à l'égard des traitements insecticides pour lutter contre les ravageurs a profondément affecté les activités des industries et réduit leur compétitivité. Les industries ont été accusées par les agriculteurs de ne pas leur donner des insecticides et d'autres produits de qualité. «Nous [les industries] avons été accusés par les agriculteurs que nos insecticides n'étaient pas assez bons » (interview). Les industries ne pouvaient pas réaliser des profits élevés avec le coton conventionnel infesté. Compte tenu de la situation, il est devenu vital pour elles de trouver une alternative appropriée à leurs problèmes économiques et sociaux. La technologie Bt qui est censée contrôler l'infestation par les insectes serait acceptable pour ce groupe social car elle pourrait les aider à retrouver leur réputation mondiale. En effet, « cette technologie a permis à certains pays de faire face au phénomène de résistance et de regagner leur compétitivité » (YAMEOGO 2011 : $11)$.

\subsection{Les chercheurs en coton}

Parmi les acteurs impliqués dans la culture du coton conventionnel, les chercheurs ont joué un rôle important dans leur quête pour comprendre ce qui se passait afin de proposer des solutions adéquates aux différents problèmes rencontrés par le coton conventionnel. En effet, à partir de 1991, l'INERA, le centre de recherche agricole a identifié des recherches de laboratoire et de terrain pour résoudre les problèmes auxquels étaient confrontés les agriculteurs, l'industrie et l'ensemble du pays. La raison de la chute de la production était une combinaison de climat irrégulier, de dégradation des sols et de dommages causés par les insectes. En 1997, les chercheurs ont découvert que le problème qui affectait le coton conventionnel était la "résistance ». Après une longue période d'utilisation des mêmes insecticides, les insectes s'y sont habitués et y ont développé une résistance.

En outre, dans la recherche agricole, la méthode utilisée en biotechnologie était traditionnelle. Les chercheurs du Burkina Faso ne savaient pas non plus quoi que ce soit sur la biotechnologie moderne, en particulier le coton biotechnologique moderne. Par conséquent, les chercheurs peuvent être considérés comme ayant voulu apprendre à connaître cette nouvelle technologie pour aider les autres acteurs à résoudre le problème des dommages causés par les insectes du cotonnier. Par exemple, les recherches menées par (ZANGRE et MASSIMBO 2002) ont révélé l'énorme intérêt des chercheurs en biotechnologie. Selon leurs résultats, la plupart des instituts de recherche et des chercheurs ont montré un intérêt pour la biotechnologie.

\subsection{Société civile}

Le processus de la transition du coton conventionnel vers le coton biotechnologie au Burkina Faso a intégré la société civile. Pour la première fois, cette section de la société occupe une place importante dans les processus d'adoption des technologies. En effet, ce groupe ciblé peut affecter positivement ou négativement le processus et la mise en œuvre de cette innovation, en particulier le coton 
biotechnologique. Les questions environnementales, sanitaires, sociales, culturelles, économiques et éthiques sont soulevées de façon permanente dans le débat autour de cette biotechnologie et la société civile peut être préoccupée par ce débat. Ce groupe social considère la biotechnologie comme une technologie très controversée. Par conséquent, il est nécessaire de la considérer comme faisant partie du processus. Au début de la problématisation, la société civile était considérée comme l'un des principaux acteurs. Monsanto était consciente du contexte social décrit ci-dessus et de sa complexité. C'est pourquoi il a cherché à intégrer ce groupe social déjà lors de sa première réunion officielle avec des acteurs au Burkina Faso.

\subsection{Le gouvernement du Burkina Faso}

Le coton biotechnologique intéresserait certainement l'État qui aurait envisagé de devenir de plus en plus compétitif sur le marché mondial. En adoptant de nouvelles technologies, le pays obtiendrait une position de premier plan en Afrique et dans le monde (CES 2011).

La figure suivante donne une carte des différents acteurs en ce qui concerne la nécessité pour eux d'accepter la nouvelle technologie, le coton Bt. Ceci montre explicitement la problématisation de la naissance d'intérêt du groupe social concerné vers l'introduction du coton biotechnologique.

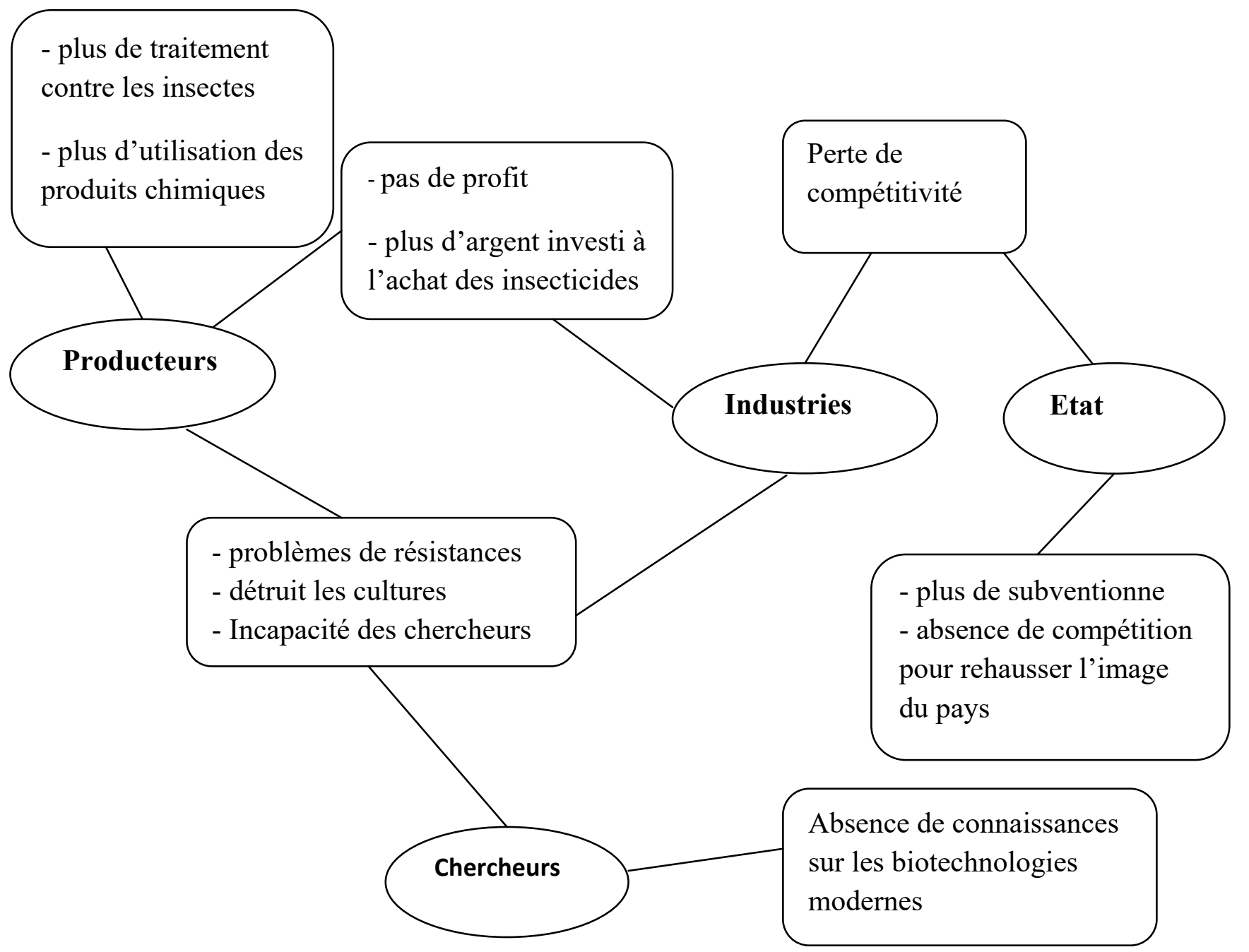

Figure 1. Acteurs et problématisation de la production cotonnière au Burkina Faso 
Par cette problématisation, les différents acteurs concernés directement par le coton biotechnologique ont été identifiés. Mais c'était encore une présomption que ces acteurs potentiels seraient intéressés par cette technologie. Le défi restant pour Monsanto était de rendre ces intérêts réels et de se placer comme un « point de passage obligatoire » selon Callon (1986). C'est-à-dire de convaincre ces acteurs concernés (agriculteurs, industries, chercheurs, société civile et gouvernement) que leurs intérêts résident dans l'admission du coton biotechnologique comme une alternative aux problèmes de productivité du coton conventionnel. Par conséquent, les deux conférences qui ont eu lieu à Ouagadougou, au Burkina Faso en 2000 et plus tard en 2003 ont été des occasions de séductions des acteurs locaux par Monsanto.

Le coton biotechnologique de Monsanto correspondait très bien aux préoccupations et aux problèmes des groupes sociaux concernés. Il s'agissait d'une solution qui permettrait aux agriculteurs et aux industries de lutter contre la résistance aux insectes sans changements majeurs, étant donné que la technologie fait partie du processus normal des espèces vivantes. Au Burkina Faso, le rendement augmentera et le nombre de traitements pesticides tombera à deux au lieu de six ou huit fois. Il a été démontré que la réduction du traitement économise du temps, des efforts et de l'argent pour les agriculteurs. L'État, serait plus compétitif sur le marché du coton. Monsanto semble alors apporter une solution au problème crucial de la résistance des insectes auquel le pays était confronté depuis les années 1990. Ceci selon Callon (1986 : 6), est considéré comme « le prémium moment de l'histoire ». En effet, les deux parties (Monsanto et les acteurs nationaux) ont traversé différentes phases qui peuvent être définies comme un processus de " traduction » selon Callon (1986). Il s'agit d'un processus au cours duquel « l'identité des acteurs, la possibilité d'interaction et les marges de manœuvre sont négociées et délimitées »(CALLON 1986 : 6). Les acteurs concernés (producteurs, industries, utilisateurs, chercheurs, décideurs, société civile, fournisseurs d'intrants chimiques) tous ont été identifiés comme des parties potentielles au Burkina Faso. En ciblant les acteurs concernés de cette manière, Monsanto, en étant le propriétaire de la technologie biotechnologique (coton Bt) remplit le rôle clé de passerelle obligatoire au sein de l'ensemble des acteurs du processus de traduction. Cela signifie que Monsanto devient indispensable dans le processus de traduction. Monsanto a affirmé avec force que sa biotechnologie cotonnière présentait des avantages économiques et environnementaux éprouvés, et elle a fait preuve d'une utilisation sûre et de produits prometteurs pour l'avenir. Dans le cas du Burkina Faso, les attentes ont joué un rôle très important dans l'adoption du coton biotechnologique. La vision particulière de la compétitivité sur le marché mondial motive l'Etat du Burkina Faso ainsi que la mobilisation des acteurs (agriculteurs, chercheurs, industries) vers l'introduction du coton biotechnologique. Ces visions particulières de l'avenir jouent un rôle clé dans la mobilisation et l'alignement des acteurs du coton au Burkina Faso. 


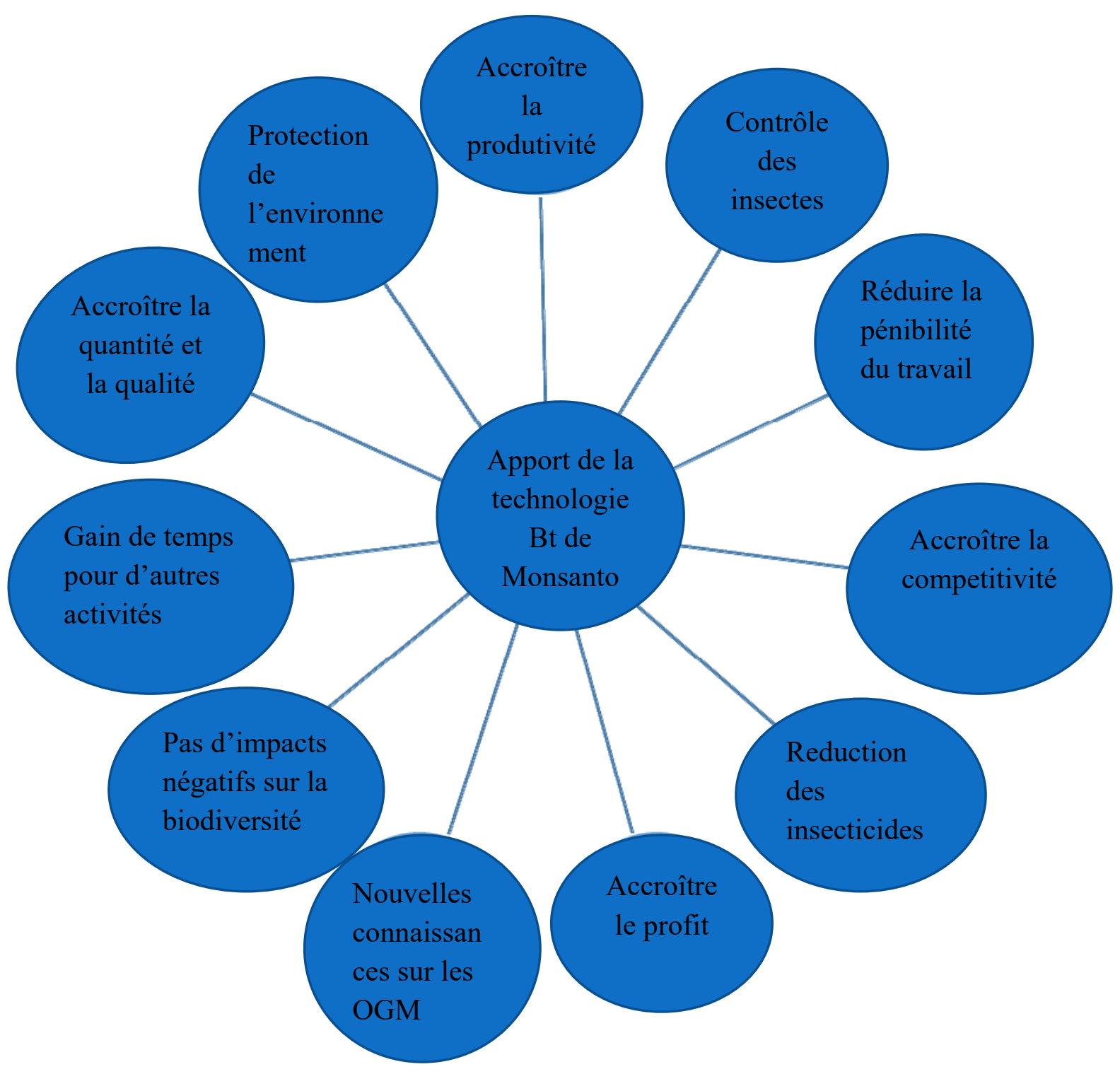

Figure 2. Avantages du coton Bt du point de vue de Monsanto : Comment Monsanto se place-t-elle comme un 'point de passage obligatoire'

Monsanto a présenté sa technologie comme une solution aux problèmes du coton conventionnel au Burkina Faso. Cela a permis d'identifier en même temps les groupes sociaux pertinents dans le processus d'innovation tel que décrit plus haut. Cependant, comme Pinch et Bijker (1986 : 35) l'affirment, " autour de chaque problème, plusieurs variantes de solution peuvent être identifiées ». Le coton biotechnologique connu sous le nom de coton Bt s'est imposé comme une solution à la lutte contre les problèmes de ravageurs du coton conventionnel. Ce coton $\mathrm{Bt}$ semblait être une des meilleures alternatives. Chaque groupe social concerné et impliqué dans la problématisation par Monsanto « peut se soumettre à l'intégration dans le plan initial, ou inversement, refuser la transaction en définissant son identité, ses objectifs, ses projets, ses orientations, ses motivations ou ses intérêts d'une autre manière » (CALLON $1986: 8)$. Cependant, avant tout véritable « intéressement », nous entendons « une série de processus par lesquels les chercheurs cherchaient à enfermer les autres acteurs dans les rôles qui leur avaient été proposés dans ce programme » (CALLON 1986 : 1). Dans le cas de l'innovation du coton biotechnologique, les acteurs soulevaient encore des questions et des inquiétudes liées à la durabilité du coton biotechnologique dans le contexte du Burkina Faso. Cette dimension implique l'intérêt que les groupes sociaux concernés ont pour la technologie.

En effet, à partir du premier atelier tenu en 2000 à Ouagadougou, des questions liées à la santé, à la 
possibilité de nouvelles résistances aux insectes se rapportant aux problèmes de résistance des années 1990 ont été discutées. Par exemple, les agriculteurs étaient très préoccupés par leur sécurité. "Nous avons entendu dire que [GMO] rend les hommes stériles" (interview). En outre, la dépendance a également été soulevée ; l'environnement, la biodiversité et les règles nationales constituent des préoccupations des acteurs concernés par la technologie coton biotechnologique.

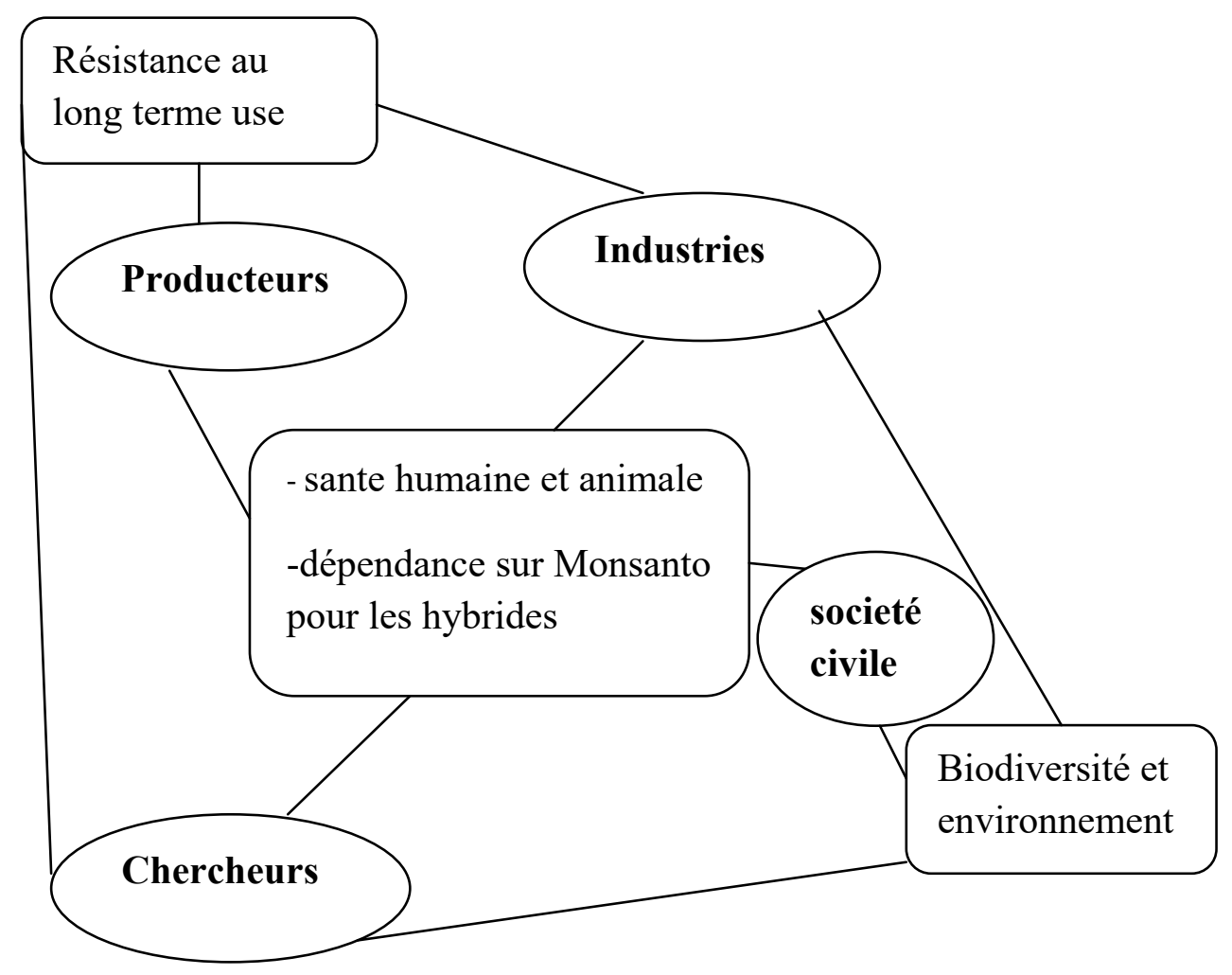

Figure 3. Émergences de préoccupations du coton Bt du point de vue des acteurs au Burkina Faso

Cela souligne la «flexibilité interprétative » de tout artefact : "Par-là, nous entendons non seulement la flexibilité dans la façon dont les gens pensent ou interprètent les artefacts, mais aussi la souplesse dans la conception des artéfacts. Il n'y a pas seulement un moyen possible ou un meilleur moyen de concevoir un artefact » (PINCH et BIJKER $1986: 40)$.

Suite à ces préoccupations, Monsanto avec les acteurs nationaux au Burkina Faso, ont construit un dispositif qui a consisté à façonner la technologie au contexte local par son accord avec les parties intéressées. Par exemple, au lieu de transférer le gène concerné dans le coton à partir des États-Unis, Monsanto a accepté de transformer la variété locale avec la technologie Bt, réduisant ainsi la question de la dépendance dont les acteurs locaux s'inquiètent. Geels (2007) distingue trois typologies de processus dans un système technique : la reproduction, l'innovation cumulative et la transformation. Dans la typologie de la transformation, comme dans le cas du coton biotechnologique, cette rétroaction a entraîné des modifications substantielles des règles de régime et une réorientation de la trajectoire de l'innovation. Suite aux préoccupations de la société civile «outsiders »(GEELS, 2004; 2007), la rétroaction a permis à Monsanto de prendre en compte les opinions des parties intéressées et de réorienter ainsi son plan initial et son programme. Les chercheurs, la société civile étaient toujours sceptiques et auraient souhaité pousser la réflexion sur la biotechnologie plus en profondeur. Ils pensent que : 
«Le renforcement des capacités était une condition importante pour cette technologie. La formation d'abord : [...] pour l'utilisation de la technologie comment isoler l'ADN et l'introduire. Maintenant ce qui manquait, [...] [était] ce renforcement des capacités, [...] à la fin de l'expérimentation, nous avons besoin d'avoir des terrains, des infrastructures, un laboratoire avec un équipement adéquat qui nous permettra de couper l'ADN et de le coller $»$ (interview).

Pour ce participant, il était important de renforcer la capacité de recherche locale afin d'accueillir le coton biotechnologique. Cette idée a été soutenue par la société civile qui a estimé que la capacité scientifique de faire de telles recherches doit être renforcée avant que des décisions puissent être prises. Cependant, Monsanto a opté de travailler surtout avec des acteurs individuels parmi les industries cotonnières, les producteurs et les chercheurs qui étaient prêt à défendre la technologie.

\section{La stabilisation du processus d'adoption du coton Bt}

Il y a donc une stabilisation relative de l'artefact puisque Monsanto a réussi à répondre à certaines des exigences des groupes sociaux concernés. Callon (1986) appelle cette rétroaction des relations qui façonnent et alignent Monsanto avec les acteurs concernés au Burkina Faso « le triangle de l'intéressement ». Les essais se sont terminés par une proposition pratique de $80 \%$ de Bt contre $20 \%$ de 'zone refuge' pour le coton conventionnel pour aider à lutter contre la résistance des insectes. Une augmentation du rendement en moyenne de $35 \%$, jusqu'à $48 \%$, dans les variétés locales contenant Bollgard II Une réduction du nombre de traitements par champ de six passes à deux fois. Une réduction de l'utilisation des pesticides et du nombre de traitements permet d'économiser $(20 €)$ par hectare, ce qui représente une réduction des coûts de 62\% (CNRST, 2007). Les autres acteurs concernés, tels que les gros agriculteurs et les industries, étaient des spectateurs amusés attendant un verdict final; Ils étaient tout à fait prêts à accepter les conclusions des spécialistes du cotonnier que sont les chercheurs.

A cette étape, les acteurs, à l'exception de la société civile, ont été apparemment séduits mais certain restèrent sceptiques. L'argument général est qu'ils ont été vraiment impressionnés par la technologie de Monsanto. Geels (2007) a qualifié ce long processus de processus de "transformation 》 où les commentaires, les modifications et les conclusions sont faits. La plupart des différents acteurs alliés affirment que le problème de ravageur est résolu, mais la société civile et certains chercheurs sont restés immuables quant à ces croyances sur la technologie. Cette attitude rend la stabilisation en attente.

Les dispositifs d'intérêts de quelques acteurs, créent un certain «équilibre de pouvoir favorable » (CALLON 1986). Pendant sept ans, le coton Bt fut commercialisé avec apparemment du succès, des structures régionales comme le NEPAD - ABNE (African Biosafety Network of Expertise) s'installèrent au Burkina Faso. Le pays fut alors le premier pays africain à commercialiser le coton biotechnologique ou encore coton Bt sur le marché mondial. Ce fut une sorte d'apogée significative, mais qui n'a que trop duré car elle a été chimérique.

\section{Discussion et conclusion sur l'échec du coton Bt au Burkina Faso}

Cet article a examiné la mise en forme de la technologie Bt dans le secteur du coton au Burkina Faso. Il a analysé le processus de transition du coton conventionnel au coton biotechnologique et a mis en évidence les différents " moments » dans ce processus. Les contraintes internes de la productivité dans le système sociotechnique du coton conventionnel d'une part, et les promesses extérieures de la 
biotechnologie développées par Monsanto pour résoudre ces problèmes d'autre part constituaient un contexte favorable pour le coton biotechnologique.

Dans la trajectoire de l'innovation du coton biotechnologique au Burkina Faso, des éléments fondamentaux doivent être saisis et servir de leçon pour toutes autres innovations. Sur la base de raisons pertinentes (efficacité, gain, connaissance), certains ont accepté la technologie $\mathrm{Bt}$ ou coton biotechnologique au Burkina Faso, d'autres par contre se basent sur des raisons éthiques (dérèglement de la nature humaine et non humaine) pour rejeter cette innovation. En plus, certains ont soulevé les possibilités de dépendance du pays vis avis du multinational (Monsanto). Ils sont donc sceptiques face à l'absence de garantie sur ses effets néfastes sur l'homme et la nature. Cependant, en 2009, le coton Bt fut commercialisé. Il a apparemment connu du succès dans le contexte burkinabè. En général, la plupart des avantages sont limités à une poignée d'acteurs, à savoir les grands acteurs industriels et Monsanto, tandis que le grand nombre d'agriculteurs qui ont besoin d'une technologie sûre et réussie qui améliorerait leurs conditions de vie grâce à une augmentation des bénéfices économiques et sociaux voient leur situation se détériorer.

En début 2016, le réseau des acteurs (1'Association Inter Professionnelle des Producteurs du Coton du Burkina (AICB) composée de chercheurs, de producteurs et d'industries d'une part et d'autre part Monsanto) de l'innovation coton biotechnologique se 'disloque'. Les acteurs l'AICB et Monsanto autrefois en symbiose s'accusent laissant place aux débats et commentaires de tous genres. Comme le soutient Fok, $(2006,2016)$ la trajectoire de l'innovation coton biotechnologique au Burkina Faso montre une certaine précipitation qui n'a pas permis un temps assez suffisant pour effectuer les recherches croisées dans le but d'avoir une variété de qualité et des normes internationales du marché. « Je vous dis que le croisement n'a pas suivi les normes afin d'avoir une bonne variété compétitive sur le marché » (interview).

En fait, la réalité est qu'aujourd'hui, on sait que ce n'est pas l'argument scientifique qui a seulement entrainé l'adoption du coton biotechnologique au niveau du Burkina. Il y a eu la pression politique, la corruption, l'ironie des acteurs nationaux pour des questions financières, le mensonge. Le coton biotechnologique a été suspendu pour ne pas dire abandonné en raison de l'incapacité à évaluer ex anté les risques économiques et sociaux qui se présentaient. Ce sont des pratiques qui rattrapent toujours le processus de l'innovation, car l'innovation progresse à l'épreuve du terrain. Les actions qui ont contribué à exclure des acteurs qu'il ne fallait pas exclure, à contourner des situations qu'il ne fallait pas contourner ont fait que l'innovation a souffert du résultat d'une négociation entachée du rapport de pouvoir du réseau d'acteurs. Ce qui ressort est que le besoin d'une nouvelle technologie est défini en termes de niches, les besoins le niveau d'accès, la représentativité et le pouvoir des acteurs dans le réseau à s'imposer.

Qui parle au nom de qui ? Qui représente qui ? Ces questions cruciales doivent être répondues si l'innovation biotechnologique veut réussir. Dans le cas du Burkina Faso, l'interprétation et l'enrôlement concernent quelques individus. Les acteurs impliqués dans le processus d'adoption du coton biotechnologique ont-ils joué un rôle représentatif? Lorsque Monsanto négocie avec le syndicat national des représentants des agriculteurs et des chercheurs, il considère que cela est représentatif de tous les agriculteurs et de tous les chercheurs. Le feu vert pour le coton Bt n'est pas donné par les chercheurs et / ou les agriculteurs, mais par leurs représentants. Ce sont ceux qui permettent les essais et accueillent la nouvelle technologie au nom des autres. Dans un processus d'innovation c'est un point crucial pour la pérennisation de l'innovation. Au début de 2016 au bout de sept ans de commercialisation le coton $\mathrm{Bt}$ a été officiellement abandonné en raison du manque à gagner en quantité de fibres produites, de la réduction de la longueur de la fibre (DOWD-URIBE et SCHNURR, 2016, FOK 2016).

Ainsi, le Burkina Faso qui était le pays pionnier en développement qui s'est lancé dans la production du coton transgénique en Afrique de l'Ouest a décidé de retourner cette année au coton conventionnel. 
L'industrie burkinabè tente au regard des pertes économiques constatées sur les marchés internationaux de poursuivre juridiquement Monsanto pour un dédommagement estimé à 84 millions de dollars. Cet état de fait nous interpelle sur le besoin de réguler les stratégies d'investissements privés dans les pays du Sud au regard de leurs impacts potentiels sur le développement dans le cadre de dispositifs partenariaux non uniquement instrumentés par les firmes privées. Il interroge certes la pertinence des méthodes d'évaluation de résultats et d'impacts mobilisables mais également les conditions institutionnelles (COMPAORE 2015; TEMPLE et al. 2016) dans le contexte des pays en développement. A un autre niveau, cet échec révèle comment la recherche scientifique privée reste enfermée dans la trajectoire technologique posée par le modèle productif d'accroissement des rendements dans l'agriculture qui l'a conduit à rechercher en continu des solutions aux impasses techniques qu'elle rencontre mais sans chercher à changer de paradigme social et technologique (TEMPLE et al., 2015, COMPAORE 2015, 2016, TOUZARD et al. (2015). Ainsi les justifications portées par la mise au point du coton biotechnologique sont-elles essentiellement le besoin de contourner les résistances occasionnées par les anciennes molécules chimiques provenant des insecticides? En aucune façon le processus d'adoption de ce coton n'a interrogé de façon explicite les besoins d'envisager un changement du modèle social qui gouverne le changement technique et organisationnel dans l'agriculture du sud depuis la révolution verte. Les progrès scientifiques et technologiques ne sont pas nécessairement une panacée pour les problèmes de développement et, dans certains cas, peuvent aggraver la pauvreté (JOLY 2001, COMPAORE 2015). Tout débat sur le rôle de la science et de la technologie au service du développement doit tenir compte de la structure des dispositifs sociotechniques dans lesquels ces ressources doivent être affectées.

\section{Bibliographie}

AKRICH M., et al., "A quoi tient le succès des innovations? 1: L'art de l'intéressement; 2: Le choix des porte-parole". Gérer et comprendre, Annales des mines, 1988.

BAFFES J., "Distortions to cotton incentives in Bénin, Burkina Faso, Chad, Mali and Togo." World Bank research working paper. Washington, DC: World Bank, 2007.

BORLAUG N. E., "Ending world hunger. The promise of biotechnology and the threat of antiscience zealotry." Plant Physiology124(2): 487-490, 2000.

CALLON M., Some elements of a sociology of translation; domestication of the scallops and the fishermen of St Brieuc Bay. In Law J. (ed.), Power, Action and Belief. A New Sociology of Knowledge? Routledge and Kegan Paul, London, 1986.

CALLON M., Techno-economic networks and Irreversibility. In Law, J. (ed.), A Sociology of Monsters. Essays on Power, Technology and Domination, Routledge, London, 1991.

CALLON M. and O. Blackwell O., "Actor-Network Theory." The Politics of Interventions: 273. Carlsson, B. (2006). "Internationalization of innovation systems: A survey of the literature." Research Policy 35(1): 56-67, 2007.

CES, "Biotechnology et le Development Economic du Burkina Faso, Burkina Faso." Premiere Session extra-ordinaire 28 Mars- 19 Avril 2011.

CNRST, "Preparation du plan startegic de la recherech scientific." Burkina Faso. Ouagadougou: Ministère des Enseignements secondaire, supérieur et de la Recherche scientifique, rapport des travaux, 1994.

CNRST, "Plan startegic de la reherche scientific au Burkina Faso, ." Ouagadougou: Ministère des Enseignements secondaire, supérieur et de la Recherche Scientifique, CNRST, Dossier, Spécial «Plan Stratégique », EURÊKA no 26/27, 1995.

CNRST, "Production cotonnière au Burkina Faso, La Recherche, un Maillon Essentiel'." Revue Eureka, 2007.

COMPAORÉ M. F. W E., The role of the National Innovation Systems Framework in facilitating socio-economic development in Burkina Faso: model and policy practice. $\mathrm{PhD}$ thesis, University of Nottingham., $\mathrm{PhD}$ thesis, University of Nottingham,

http://eprints.nottingham.ac.uk/36975/1/PhD\%20thesis\%20final\%20MFW\%20Eveline\%20Compaore..pdf, 2015. 
COMPAORE SAWADOGO E. M.F.W. 'Transition Politique et Accumulation Capitaliste : Le Cas du Commerce du Coton au Burkina Faso Post Révolutionnaire', in Sanou F. and Sissao A.J. Développement Endogène de l'Afrique et Mondialisation : Une Relecture de la Pensée du Professeur Joseph Ki-Zerbo, Presse Universitaire de Ouagadougou : Fondation Joseph Ki-Zerbo, 2016.

DOWD-URIBE B. AND SCHNURR M., A., "Briefing: Burkina Faso's reversal on genetically modified cotton and the implications for Africa". African Affairs 1 (12) [First published online], 2016

EZEZIKA O. C., et al., "The value of trust in biotech crop development: a case study of Bt cotton in Burkina Faso." Agriculture \& Food Security1(Suppl 1): S2, 2012.

FOK M., Liberalization and globalization: Trojan Horse for the cotton traders' domination in Francophone Africa. International Association of Agricultural Economists Conference, 2006.

FOK M., «Impacts du coton-Bt sur les bilans financiers des sociétés cotonnières et des paysans au Burkina Faso ». Cahiers Agricultures, 25 (3), 2016.

GEELS F. W., "From sectoral systems of innovation to socio-technical systems: Insights about dynamics and change from sociology and institutional theory." Research Policy 33 (6), pp. 897-920, 2004.

GEELS F. W. AND SCHOT J., "Typology of sociotechnical transition pathways." Research Policy 36 (3), pp 399-417, 2007.

GRAY L. C., "Is land being degraded? A multi scale investigation of landscape change in southwestern Burkina Faso." Land Degradation \& Development 10 (4), pp 329-343, 1999.

GRAY L. C., "What kind of intensification? Agricultural practice, soil fertility and socioeconomic differentiation in rural Burkina Faso." The geographical journal 171 (1),p. 70 82, 2005.

GRAY C. L., Cotton Production in Burkina Faso, International Rhetoric Versus Local Realities,'In Moseley G. W. and Gray L. (eds) Hanging by a thread: Cotton, globalization, and poverty in Africa, Athens, Ohio: Ohio University Press, 2008.

GROSS R., "World Intellectual Property Organisation (WIPO)." GISWatch: Global Information Society Watch: 65-73, 2007.

ILLY AND ZANGRE R., "les Nouvelles Technologies et Development Agricole." CNRST : MESSRS, 2003.

ISAAA. "Journées portes ouvertes des stations expérimentales du coton Bt au Burkina Faso, Bt Cotton Trials in Burkina Faso." 13-16 November, 2006. http://www.isaaa.org/resources/videos/burkinafaso/, 2006.

ISAAA, "Increased Global Adoption of Biotech Crops: Advancing best practice in biotechnology." ISAAA Reports (2) 1, 2012.

JAMES C., Global Status of Commercialized Biotech/GM Crops, 2009, ISAAA Ithaca, NY, 2009.

JOLY P B ., Les OGM entre la Science et le Public? Quatre Modèles pour la gouvernance de l'Innovation et des risques. Economie Rurale (226), p 11-29, http://www.persee.fr/doc/ecoru_0013-0559_2001_num_266_1_5273, 2001.

LATOUR B., La Science en Action : Introduction à la Sociologie des Sciences. Paris : la Découverte.

Monsanto, Leading in innovation: 2011 annual report, Forbes Magasine, http://www.monsanto.com/investors/pages/archived-annual-reports.aspx, 2011.

PINCH TREVOR J. AND WIEBE BIJKER E., "Science, Relativism and the New Sociology of Technology: Reply to Russell." Social Studies of Science 16: 347-360, 1986.

QAYUM A., AND SAKKHARI K., "Bt cotton in Andhra Pradesh." A three-year assessment. The first ever sustainabled independent scientific study of Bt cotton in India. Booksline, Hyderabad, india., 2005.

ROBERTS R, "The Coercion of Free Market: Cotton, Peasants and the Colonial State in the French Soudan, 1924-1932'." Isaacman and Roberts, eds.,_Cotton, colonialism, p. 231-243, 1995.

ROBIN M. M., The World according to Monsanto, Documentary, 1h49.01, http://www.gmwatch.org/gm-videosb/6-mustsee-videos/11673-the-world-according-to-monsanto [access date 10/08/2011], 2008.

SCHWARTZ A., 'L'exploitation agricole de l'aire cotonnière burkinabè : Caractéristiques sociologiques, Démographiques, Economiques', Document de Travail, ORSTOM, Ouagadougou, 1991.

SCHWARTZ A., Attitudes to cotton growing in Burkina Faso: Different farmers, different behaviours. In Cattin B.M, Griffpn B. and Guillaumont P. (eds) Economics of agricultural policies in developing countries, Paris: Revue Française d'Economie, 1995. 
SCHWARTZ A., Des temps anciens à la dévaluation du franc CFA, les tribulations de la culture du coton au Burkina Faso. Annales de géographie, JSTOR, 1997.

SCHWARTZ A., "La politique cotonnière du gouverneur Hesling et la dislocation de la colonie de Haute-Volta en 1932: et si l'inspecteur Sol s' était trompé?". In Madiega G. Y. et Nao O (Eds) Burkina Faso Cent Ans d'Histoire, 18951995, Paris: Karthala, 1587-1623, 1999.

SHIVA V., The Role of Patents in the Rise of Globalization Interview was conducted by Nic Paget-Clarke on August 27, 2003 for In Motion Magazine in New Delhi, India http://www.inmotionmagazine.com/global/vshiva4_int.html, 2003.

SMITH A., et al., "The governance of sustainable socio-technical transitions" Research Policy 34 (10), p 1491-1510, 2005.

TEMPLE L., TOUZARD JM., BOYER J., REQUIER DESJARDINS D., Comparaison des trajectoires d'innovation pour la sécurisation alimentaire des pays du Sud. Revue,_Biotechnol. Agron. Soc. Environ. 19 (1), 53-61. http://popups.ulg.ac.be/1780-4507/index.php?id=11824, 2015.

TEMPLE L., BIÉNABE E., BARRET D. \& SAINT-MARTIN G., Methods for assessing the impact of research on innovation and development in the agriculture and food sectors, African Journal of Science, Technology, Innovation and Development, DOI: 10.1080/20421338.2016.1219484. http://dx.doi.org/10.1080/20421338.2016.1219484, 2016.

TIENDREBEOGO O., 'Burkina Faso is a Trojan horse for GMOs in Africa', Journal of Alternatives, June 282011 http://gmwatch.org/latest-listing/1-news-items/13342, 2011.

TOUZARD J.M., TEMPLE L., FAURE G., TRIOMPHE B., Innovation systems and knowledge communities in the agriculture and agrifood sector: a literature review. Journal of Innovation Economics and Management, 2 (17): 117$142,2015$.

TRAORE O., "OGM et Securité Alimentaire, paper presented during workshop on Biotechnology et le Development Economic du Burkina Faso, Burkina Faso." Premiere Session extra-ordinaire 28 Mars- 19 Avril 2011.

YAMEOGO G., 'Expérimentation du Coton Bt au Burkina Faso', paper presented during workshop on Biotechnology et le Development Economic du Burkina Faso, Burkina Faso, Session extraordinaire, CES: 28 Mars- 19 Avril 2011.

ZANGRE R. AND MAZIMBO I., les Nouvelles Technologies et Développement Agricole. Ouagadougou : MESSRS/CNRST, 2002. 